Available online at www.eccomasproceedia.org

Eccomas Proceedia COMPDYN (2021) 2396-2407

ECCOMAS

$8^{\text {th }}$ ECCOMAS Thematic Conference on

Proceedia

Computational Methods in Structural Dynamics and Earthquake Engineering

M. Papadrakakis, M. Fragiadakis (eds.)

Streamed from Athens, Greece, 28 - 30 June 2021

\title{
IMPROVEMENT OF DYNAMIC AND SEISMIC BEHAVIOUR OF CABINETS THROUGH CONTROLLED DEFORMATION
}

\author{
S. Pagliaro ${ }^{1}$, A. Di Egidio ${ }^{2}$, and A. Contento ${ }^{3}$ \\ ${ }^{1}$ University of L'Aquila - DICEAA \\ via G. Gronchi 19, 64100 L'Aquila - Italy \\ e-mail: stefano.pagliaro@graduate.univaq.it \\ ${ }^{2}$ University of L'Aquila - DICEAA \\ via G. Gronchi 19, 64100 L'Aquila - Italy \\ e-mail: angelo.diegidio@univaq.it \\ ${ }^{3}$ University of L'Aquila - DICEAA \\ via G. Gronchi 19, 64100 L'Aquila - Italy \\ e-mail: alessandro.contento@univaq.it
}

\begin{abstract}
In this paper the possibility to improve the dynamic and seismic response of cabinets that can exhibit rocking dynamics is investigated. The generic cabinet is modelled as an assembly of rigid components and can undergo a change of configuration. The scope of the research is to verify if the allowed deformation could protect the cabinet by preventing the overturning or reducing the amplitude of rocking oscillations. The control of the deformation is obtained by using linear visco-elastic devices, whose mechanical characteristics are at the base of the parametric analysis performed in the paper. An extensive parametric analysis is carried out by numerically integrating the equations of motion. The effectiveness of this protection method is analyzed by comparing the behaviour of rigid and deformable cabinets under one-sine pulse and seismic excitations. For the one-sine pulse excitation, the comparison is made with overturning spectra that provide the amplitude of the excitation able to overturn the cabinets versus its circular frequency. For the seismic excitation, the comparison is performed by using different registered earthquakes and comparing the maximum rocking angle reached during the motion. The results show that the ability of cabinets to change their configuration improves the dynamical and seismic performances compared to those of the equivalent rigid cabinets.
\end{abstract}

Keywords: Deformable rocking model, overturning, rocking maps, one-sine impulse, seismic excitation. 


\section{Introduction}

The protection of rigid blocks from the overturning is a fascinating argument in the scientific literature. Most of the studies on the protection of rigid blocks from overturning are focused on the use of external devices such as base isolation $[1,2,3,4,5]$ and base anchorages [6, 7]. Several authors used mass-dampers, that have been modeled either in the shape of a pendulum $[8,9,10,11]$ or as a single degree of freedom mass running on the top of the rigid body $[12,13$, 14]. Some researchers studied the use of semi-active anchorages $[15,16]$ or base active control systems $[17,18,19]$ to increase the amplitude of base excitation required to topple a rigid body.

This paper investigates the dynamic and seismic response of cabinets that are constituted by four external panels, hinged to each other in the four vertexes of the body. Four linear viscoelastic devices of equal characteristics are associated with these hinges. Due to this particular configuration, the body can deform as an articulated quadrilateral. Additionally, it is assumed that the body can undergo a rocking motion but cannot slide. The equations of motion are obtained by a Lagrangian approach. Two different impacts are considered in the paper: the classical impact of the base of the body with the support during the rocking motion and the impact on security stops, needed to limit the maximum angular deformation.

The model presented here is conceptually similar to the flexible rocking body models presented in $[20,21,22]$ or [23], where the flexible rocking model was used to interpret the experimental response of tuff walls. However, the novelty of the paper is not in the description of a flexible rocking structure but in the investigation of the behaviour of a structure where specific devices are added to guide its deformation with the aim of reducing the amplitude of the rocking oscillation and preventing the overturning.

An extensive parametric analysis is performed by numerically integrating the equations of motion. The effectiveness of this protection method is analyzed by comparing the behaviour of rigid (i.e., with fixed connections) and deformable cabinets under one-sine pulse and seismic excitations. For the one-sine pulse excitation, the comparison is made with overturning spectra that provide the amplitude of the excitation able to overturn the cabinets versus its circular frequency. For the seismic excitation, the comparison is performed by building rocking maps that are contour plots of the maximum rocking angle in a specific parameter plane. Different recorded earthquakes have been used in the simulations.

\section{Mechanical model of the cabinet}

The mechanical system used to schematize the cabinet is shown in Fig. 1a. The perimeter panels are connected through hinges and four equal Kelvin-Voight devices in the vertexes of the cabinet (see Fig. 1a). With this particular structure, the mechanical system can deform as an articulated quadrilateral and its kinematics would be undetermined without the Kelvin-Voight devices. The vertical distance $h$ between two consecutive shelves is constant so the total height of the cabinet is $4 h$. The base of the cabinet has a dimension $2 b$ and its depth (i.e., the dimension orthogonal to the plane of Fig. 1) is $d=1.0 \mathrm{~m}$.

All panels have a thickness $s=0.02 \mathrm{~m}$ and a bulk density $\rho=650 \mathrm{~kg} / \mathrm{m}^{3}$ (i.e., chipboard wood). The cabinet may contain additional masses placed on the shelves. Such masses are quantified multiplying a bulk density $\rho_{\text {fill }}=130 \mathrm{~kg} / \mathrm{m}^{3}$ for the volume between two consecutive horizontal panels and are added to the mass of the panel on which they are placed. Considering also the additional masses $M$, the masses of the seven panels $m_{j}(j=1, \ldots, 7)$ are evaluated as 


$$
\begin{aligned}
& m_{1,3}=\rho \cdot d \cdot s \cdot 4 h \\
& m_{2,4-7}=\rho \cdot d \cdot s \cdot 2 b+M
\end{aligned}
$$

It is assumed that the additional mass $M$ is located in the same mass centre of the panel on which it is placed and that there is no inertia moment associated to these masses. Since the sliding of the cabinet is prevented, the motion of the body can be divided into two different phases: $(i)$ the full-contact phase, where the body deforms as an articulated quadrilateral (Fig. 1b), and (ii) the rocking phase, where the body rotates alternatively around one of its base corners and deforms at the same time (Fig. 1c). The Lagrangian parameter $\theta(t)$ describes the deformation of the cabinet, whereas the parameter $\psi(t)$ describes the rocking motion.

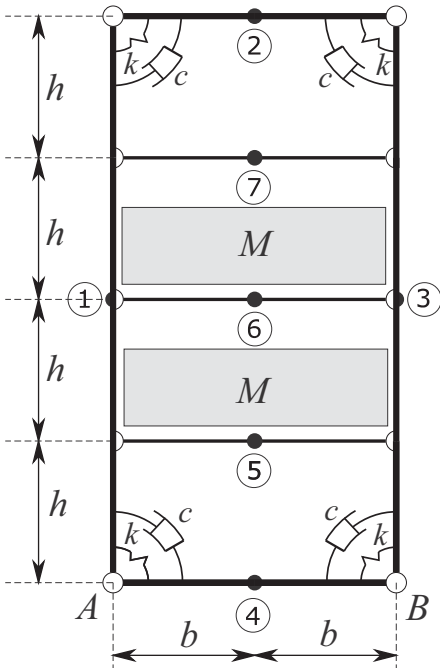

(a)

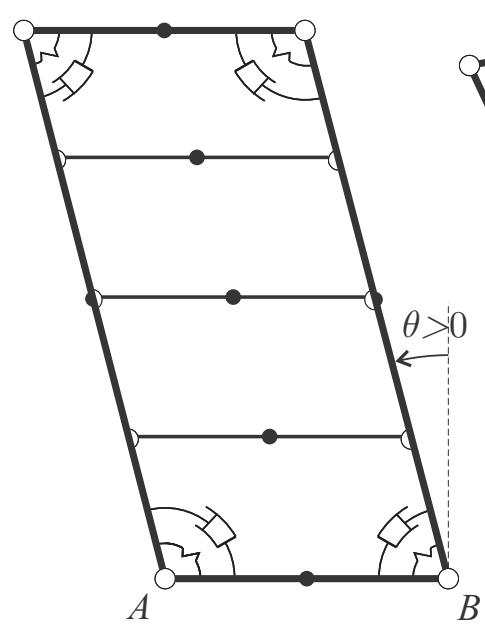

(b)

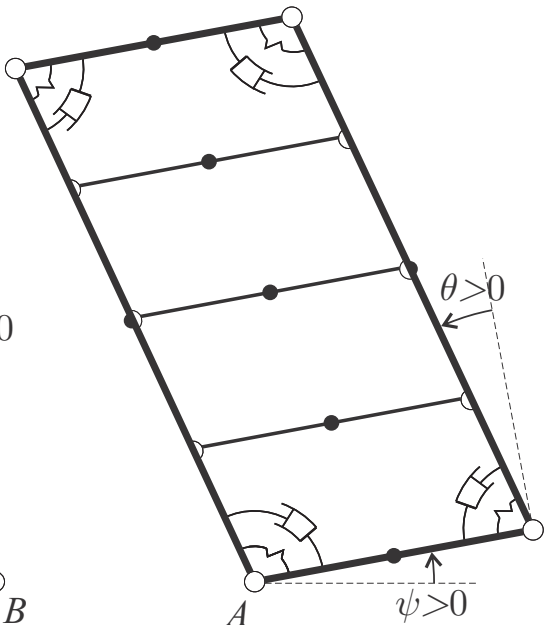

(c)

Figure 1: Model: (a) Geometrical characterization; (b) Full-contact deformed configuration; (c) Rocked and deformed configuration.

\subsection{Equations of motion}

The equations of motion are obtained through a Lagrangian approach. For brevity, only the equations of the rocking phase around the left corner $A$ are presented in this section. The equations of the full-contact phase can be obtained by those of the rocking phase by neglecting the angle $\theta(t)$ and its time-derivatives $(\theta(t)=\dot{\theta}(t)=\ddot{\theta}(t)=0)$. The two equations of motion of the body that rocks around the left base corner read:

$$
\begin{gathered}
h^{2}\left(4 m_{1}+16 m_{2}+4 m_{3}+m_{5}+4 m_{6}+9 m_{7}\right)\left[\psi^{\prime \prime}(t)+\theta^{\prime \prime}(t)\right]- \\
b h\left(4 m_{2}+4 m_{3}+m_{5}+2 m_{6}+3 m_{7}\right)\left\{\psi^{\prime \prime}(t) \sin [\theta(t)]-\psi^{\prime}(t)^{2} \cos [\theta(t)]\right\}- \\
h\left(2 m_{1}+4 m_{2}+2 m_{3}+m_{5}+2 m_{6}+3 m_{7}\right) g \sin [\theta(t)+\psi(t)]- \\
h\left(2 m_{1}+4 m_{2}+2 m_{3}+m_{5}+2 m_{6}+3 m_{7}\right) x_{g r}^{\prime \prime}(t) \cos [\theta(t)+\psi(t)]+ \\
\left(J_{1}+J_{3}\right) \theta^{\prime \prime}(t)+4 c \theta^{\prime}(t)+4 k \theta(t)=0
\end{gathered}
$$




$$
\begin{gathered}
\left(J_{1}+J_{2}+J_{3}+J_{4}+J_{5}+J_{6}+J_{7}\right) \psi^{\prime \prime}(t)+ \\
b^{2}\left(m_{2}+4 m_{3}+m_{4}+m_{5}+m_{6}+m_{7}\right) \psi^{\prime \prime}(t)- \\
2 b h\left(4 m_{2}+4 m_{3}+m_{5}+2 m_{6}+3 m_{7}\right) \psi^{\prime \prime}(t) \sin [\theta(t)]- \\
2 b h\left(4 m_{2}+4 m_{3}+m_{5}+2 m_{6}+3 m_{7}\right) \psi^{\prime}(t) \theta^{\prime}(t) \cos [\theta(t)]+ \\
h^{2}\left(4 m_{1}+16 m_{2}+4 m_{3}+m_{5}+4 m_{6}+9 m_{7}\right)\left[\psi^{\prime \prime}(t)+\theta^{\prime \prime}(t)\right]+ \\
b\left(m_{2}+2 m_{3}+m_{4}+m_{5}+m_{6}+m_{7}\right)\left\{g \cos [\psi(t)]-\sin [\psi(t)] x_{g r}^{\prime \prime}(t)\right\}- \\
b h\left(4 m_{2}+4 m_{3}+m_{5}+2 m_{6}+3 m_{7}\right)\left\{\theta^{\prime \prime}(t) \sin [\theta(t)]+\theta^{\prime}(t)^{2} \cos [\theta(t)]\right\}- \\
h\left(2 m_{1}+4 m_{2}+2 m_{3}+m_{5}+2 m_{6}+3 m_{7}\right) g \sin [\theta(t)+\psi(t)]- \\
h\left(2 m_{1}+4 m_{2}+2 m_{3}+m_{5}+2 m_{6}+3 m_{7}\right) x_{g r}^{\prime \prime}(t) \cos [\theta(t)+\psi(t)]=0
\end{gathered}
$$

Eq. 2 models the deformation of the body and Eq. 3 models the rocking motion. In the remaining of the paper, the dependence of $\theta, \psi$, and $x_{g r}$ on $t$ is omitted.

\subsection{Uplift conditions}

The uplift acceleration $a_{U P}^{A}$, able to uplift the body around $A$ coincides with the expression of $x_{g r}^{\prime \prime}$ obtained from Eq. 3 when $\psi=\dot{\psi}=\ddot{\psi}=0$

$$
\begin{gathered}
\mathrm{a}_{U P}^{A}=\left\{b g\left(m_{2}+2 m_{3}+m_{4}+m_{5}+m_{6}+m_{7}\right)-\right. \\
b h\left(4 m_{2}+4 m_{3}+m_{5}+2 m_{6}+3 m_{7}\right)\left[\theta^{\prime \prime} \sin (\theta)-\theta^{\prime 2} \cos (\theta)\right]- \\
g h\left(2 m_{1}+4 m_{2}+2 m_{3}+m_{5}+2 m_{6}+3 m_{7}\right) \sin (\theta)+ \\
\left.h^{2}\left(4 m_{1}+16 m_{2}+4 m_{3}+m_{5}+4 m_{6}+9 m_{7}\right) \theta^{\prime \prime}\right\} / \\
{\left[h\left(2 m_{1}+4 m_{2}+2 m_{3}+m_{5}+2 m_{6}+3 m_{7}\right) \cos (\theta)\right]}
\end{gathered}
$$

The uplift acceleration $\mathrm{a}_{U P}^{A}$ depends on the geometry of the cabinet and on its deformation during the full-contact phase (i.e., depend on $\theta, \dot{\theta}$ and $\ddot{\theta}$ ).

\subsection{Impact conditions}

The model presented in this paper considers two different kinds of impact: (i) the impact between the base of the cabinet and the support during the rocking motion and (ii) the impact on security stops that are used to limit the deformation of the cabinet.

The impact conditions referring to a cabinet that after an impact re-uplifts around the left corner $A$ are obtained assuming that the cabinet position after the impact remains unchanged, the angular momentum of the whole cabinet around the re-uplifting corner $A$ is preserved, and the horizontal momentum of the cabinet is also preserved. Under such assumptions, it is possible to evaluate the post-impact angular velocities $\dot{\theta}^{+}$and $\dot{\psi}^{+}$as functions of the pre-impact angular velocities $\dot{\theta}^{-}$and $\dot{\psi}^{-}$

$$
\dot{\theta}^{+}=\dot{\theta}^{-}+r_{\vartheta}(\theta) \dot{\psi}^{-} ; \dot{\psi}^{+}=s_{\theta}(\theta)\left(\dot{\theta}^{-}-\dot{\theta}^{+}\right)+s_{\psi}(\theta) \dot{\psi}^{-}
$$

where

$$
r_{\theta}(\theta)=\frac{\left[\begin{array}{l}
4 b^{2}\left(2 m_{1}+m_{2}+m_{4}+m_{5}+m_{6}+m_{7}\right)- \\
2 b h\left(2 m_{1}+4 m_{2}+3 m_{3}+m_{5}+2 m_{6}+2 m_{7}\right) \theta
\end{array}\right]}{\left[\begin{array}{l}
b^{2}\left(m_{2}+4 m_{3}+m_{4}+m_{5}+m_{6}+m_{7}\right)- \\
b h\left(8 m_{2}+8 m_{3}+2 m_{5}+4 m_{6}+6 m_{7}\right) \theta+ \\
h^{2}\left(4 m_{1}+16 m_{2}+4 m_{3}+m_{5}+4 m_{6}+9 m_{7}\right) \theta^{2}+ \\
4 h^{2}\left(m_{1}+m_{3}\right)+J_{2}+J_{4}+J_{5}+J_{6}+J_{7}
\end{array}\right]}
$$




$$
\begin{gathered}
s_{\theta}(\theta)=\frac{h^{2}\left(16 m_{2}+m_{5}+4 m_{6}+9 m_{7}\right)+J_{1}+J_{3}}{\left[\begin{array}{l}
J_{A}-b h\left(8 m_{1}+8 m_{2}+2 m_{5}+4 m_{6}+6 m_{7}\right) \theta+ \\
h^{2}\left(4 m_{1}+16 m_{2}+4 m_{3}+m_{5}+4 m_{6}+9 m_{7}\right) \theta^{2}
\end{array}\right]} \\
s_{\psi}(\theta)=\frac{\left[\begin{array}{l}
J_{A}-2 b S_{y B}+b h\left(4 m_{1}-4 m_{3}\right) \theta+ \\
h^{2}\left(4 m_{1}+16 m_{2}+4 m_{3}+m_{5}+4 m_{6}+9 m_{7}\right) \theta^{2}
\end{array}\right]}{\left[\begin{array}{c}
J_{A}-b h\left(8 m_{1}+8 m_{2}+2 m_{5}+4 m_{6}+6 m_{7}\right) \theta+ \\
h^{2}\left(4 m_{1}+16 m_{2}+4 m_{3}+m_{5}+4 m_{6}+9 m_{7}\right) \theta^{2}
\end{array}\right]}
\end{gathered}
$$

The quantities $r_{\theta}(\theta), s_{\theta}(\theta)$, and $s_{\psi}(\theta)$ play the role of restitution coefficients and depend on the deformed configuration of the system. Inside their expressions, $J_{A}$ is the polar inertia of the whole cabinet with respect to $A$ and $S_{y A}$ is the static moment of the system with respect a vertical axis passing through $A$.

Security stops are introduced to limit the deformation (i.e., the maximum value of $\theta$ ) during the motion. By referring to an impact on security stops during a rocking motion around the base corner A, it is possible to obtain the post-impact velocities $\dot{\theta}^{+}$and $\dot{\psi}^{+}$as functions of the pre-impact angular velocities $\dot{\theta}^{-}$and $\dot{\psi}^{-}$y through the following relationships:

$$
\dot{\theta}^{+}=-\eta \dot{\theta}^{-} ; \dot{\psi}^{+}=\dot{\psi}^{-}+p(\theta, \psi) \dot{\theta}^{-}
$$

where

$$
p(\theta, \psi)=\frac{(\eta+1) h\left(2 m_{1}+4 m_{2}+2 m_{3}+m_{5}+2 m_{6}+3 m_{7}\right)}{\left[\begin{array}{c}
b\left(m_{2}+2 m_{3}+m_{4}+m_{5}+m_{6}+m_{7}\right) \sin (\psi)- \\
h\left(2 m_{1}+4 m_{2}+2 m_{3}+m_{5}+2 m_{6}+3 m_{7}\right) \theta \sin (\psi)+ \\
h\left(2 m_{1}+4 m_{2}+2 m_{3}+m_{5}+2 m_{6}+3 m_{7}\right) \cos (\psi)
\end{array}\right]}
$$

It is assumed that after an impact on security stops the value of $\dot{\theta}$ changes its sign and is reduced to a fraction of the value before the impact. The restitution coefficient $\eta$ depends on the characteristics of the security stops and $\eta \leq 1$. The angular velocity $\dot{\psi}^{+}$is obtained by assuring the conservation of the horizontal momentum of the cabinet.

\section{Numerical simulations}

An extensive parametric analysis is performed by numerically integrating the equations of motion. Such analyses are performed varying the oscillation period $T$ of the cabinet in the full-contact phase and the dimensions (i.e., base $2 b$ and slenderness $\lambda=2 h / b$ ) of the cabinet. All the other quantities are fixed: damping ratio of the cabinets $\xi=0.05$, restitution coefficient of the security stops $\eta=0.5$, maximum angle allowed by the security stops $\theta_{\max }=0.1 \mathrm{rad}$, and the cabinet is considered to be full (i.e., the additional mass $M$ is added to the mass of the shelves from 4 to 7, see Fig. 1a).

\subsection{One-sine impulsive excitation}

In the analyses performed in this section, the cabinet is excited by a one-sine pulse excitation:

$$
\ddot{x}_{g}(t)= \begin{cases}A_{s} \sin \left(\frac{2 \pi}{T_{s}} t\right) & 0 \leq t \leq T_{s} \\ 0 & T_{s}<t \leq t_{\max }\end{cases}
$$


where $T_{s}$ is the period of the one-sine cycle, $\Omega=2 \pi / T_{s}$ is the circular frequency of the excitation, $A_{s}$ is its amplitude, and $t_{\max }$ is the maximum time used in the numerical integrations $\left(t_{\max }=10 T_{s}\right)$. The results are arranged in overturning spectra, that provide the amplitude of the excitation that generates the first overturning occurrence versus the circular frequency of the excitation.

An initial parametric analysis is performed to investigate the sensitivity of the dynamics of the cabinet to $T$. In such an analysis the geometrical characteristics of the cabinet are fixed, $2 b=0.6 \mathrm{~m}, 4 h=2.6 \mathrm{~m}$. Figure $2 \mathrm{a}$ presents the results obtained with a model that does not include security stops, whereas Fig. $2 \mathrm{~b}$ shows the corresponding results obtained by including the security stops. In the two figures, dashed lines refer to a rigid cabinet, whereas solid lines refer to a deformable cabinet. The spectra are labelled with the value of the period $T$ used in the analysis. Such labels are surrounded by an ellipse if, during the motion, the cabinet impacts on security stops or by a rectangle otherwise. In Fig. $2 \mathrm{a}$, since the security stops are not considered, the labels are all rectangular. Such a convention that shows the occurrence of impacts on security stops is used in all the figures of this section. In Fig.s $2 a$ and $2 b$, the overturning spectra of the deformable cabinets with periods $T \geq 1$.0s are above the curves of the rigid cabinets in a wide range of the values of $\Omega$. This means that the amplitude of the excitation $A_{s}$ required to overturn a deformable cabinet is higher than amplitude needed to overturn the corresponding rigid cabinet. Moreover, the comparison between Fig. $2 \mathrm{a}$ and $2 \mathrm{~b}$ shows that, when impacts on security stops occur (e.g., cabinet with $T=1.5 \mathrm{~s}$ ), the presence of the security stops significantly affects the dynamics of the cabinet. Nevertheless, the overturning spectrum obtained for $T=1.5 \mathrm{~s}$ in Fig. $2 \mathrm{~b}$ is still above the curve of rigid cabinet in most of the considered range of $\Omega$. In general all the overturning spectra in Fig. 2 present a jump. As it occurs in rigid blocks $([24,25])$, also in deformable rocking bodies such a jump is associate to a change of the overturning mode. Specifically, before the jump the body overturns hitting once the ground, whereas after the jump the body overturns without hitting the ground. The deformability of the cabinet shifts the jump on the left leading to a general improvement of the behaviour of the cabinet.

In the following analysis the dimensions of the cabinet, $2 b$ and $4 h$, are considered as variable parameters. In Fig. 3, the results of the analysis are arranged in matrix form. Each row refers to a different base dimension, whereas each column refers to a different height of the cabinet. As expected, the dimensions of the cabinet significantly affect the overturning spectra of the rigid body. In general, the deformable cabinets are less prone to overturn than the equivalent rigid cabinets, especially for more slender cabinets (i.e., those in the upper row). In Fig. 3, the left graph on the first row and the middle graph on the second row refer to cabinets with $\lambda=3$ and different scale size. The comparison of the two graphs shows that the improvement in the dynamics of the deformable cabinets (solid lines) with respect to the correspondent rigid case (dashed lines) is smaller in the cabinet with larger scale.

\subsection{Seismic excitation}

A parametric analysis is performed to assess if the deformation of the cabinet can reduce the amplitude of the rocking oscillations and eventually avoid the overturning of the cabinet during a seismic event. For this analysis, seismic events with different characteristics are selected. Fig. 4 shows the time-histories of the seismic records that are listed in the following:

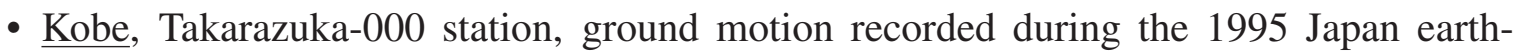
quake; 
$T=\ldots$ No impact on security stops

$T=\ldots$ Impact on security stops
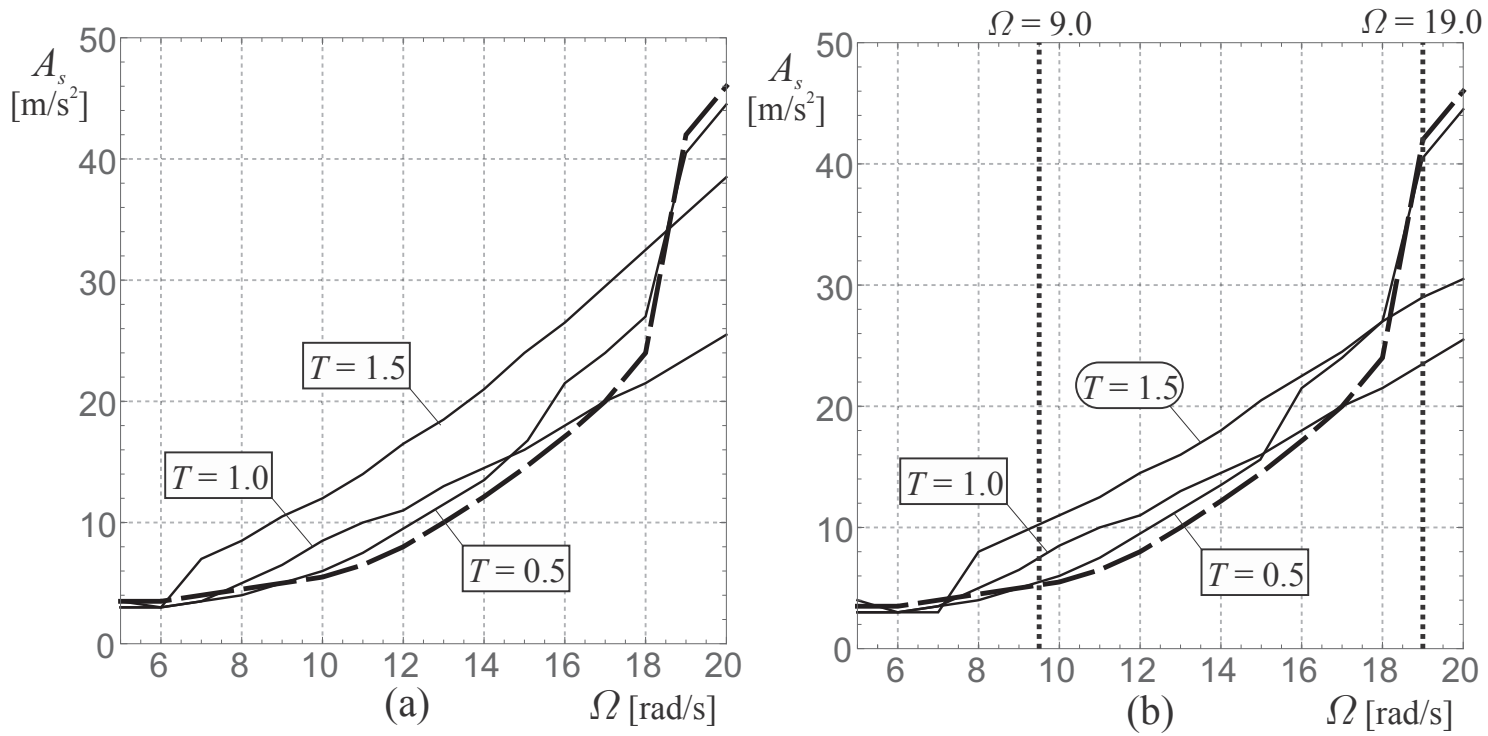

Figure 2: Overturning curves for different period $T$ : (a) Overturning curves without security stops; (b) Overturning curves with security stops $\left(\xi=0.05, \eta=0.5, \theta_{\max }=0.1,2 b=0.6 \mathrm{~m}, 4 h=2.6 \mathrm{~m}\right.$, cabinet full).

$T=\ldots$ No impact on security stops

$T=\ldots$ Impact on security stops
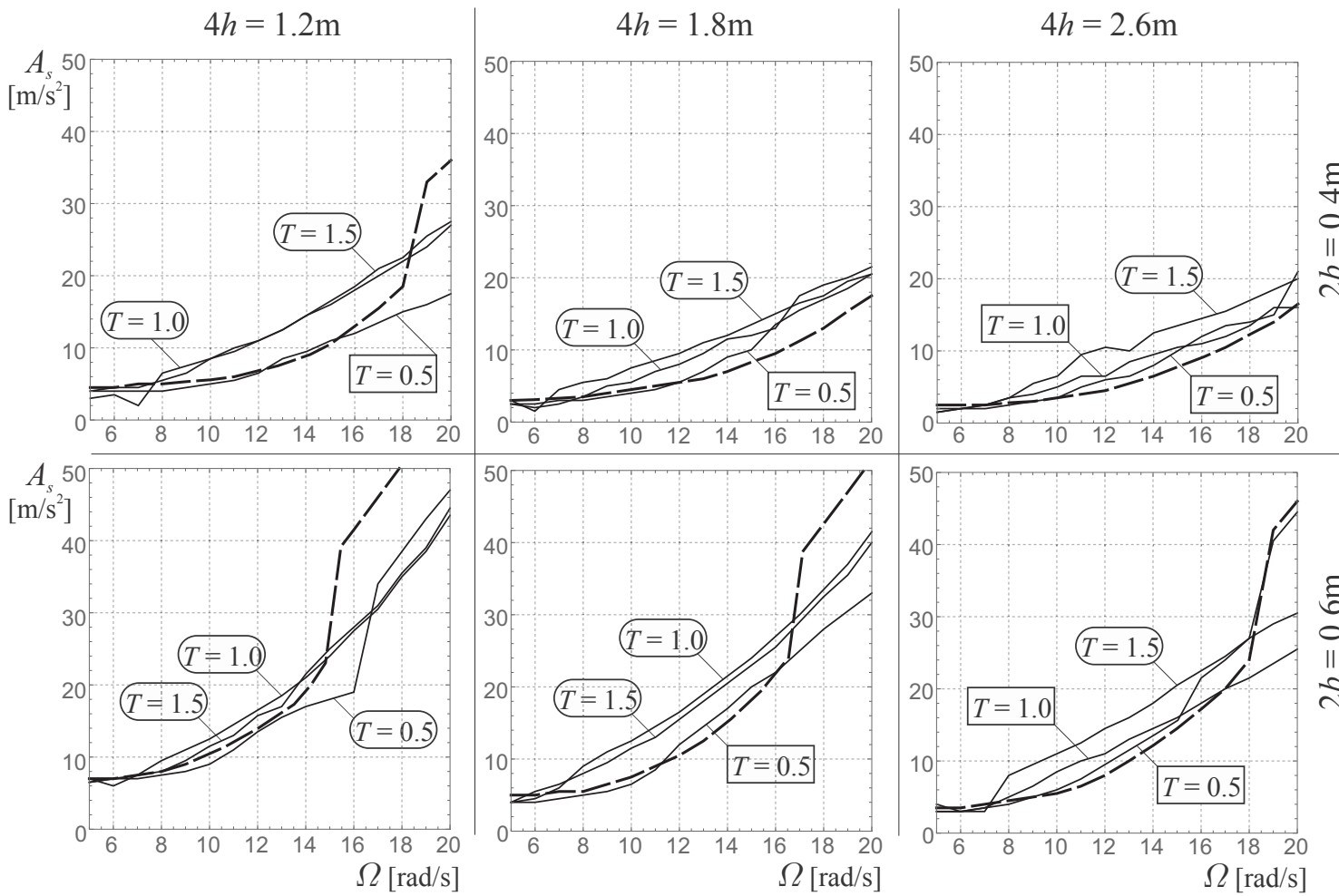

Figure 3: Overturning curve for different geometrical characteristics of the cabinet $\left(\xi=0.05, \eta=0.5, \theta_{\max }=\right.$ 0.1 , cabinet full). 
- Newhall, Newhall-360 station, ground motion recorded during the 1994 Northridge, California earthquake;

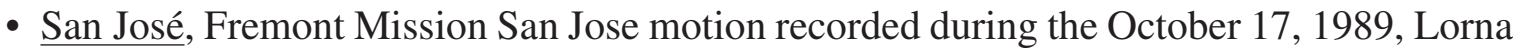
Prieta earthquake.
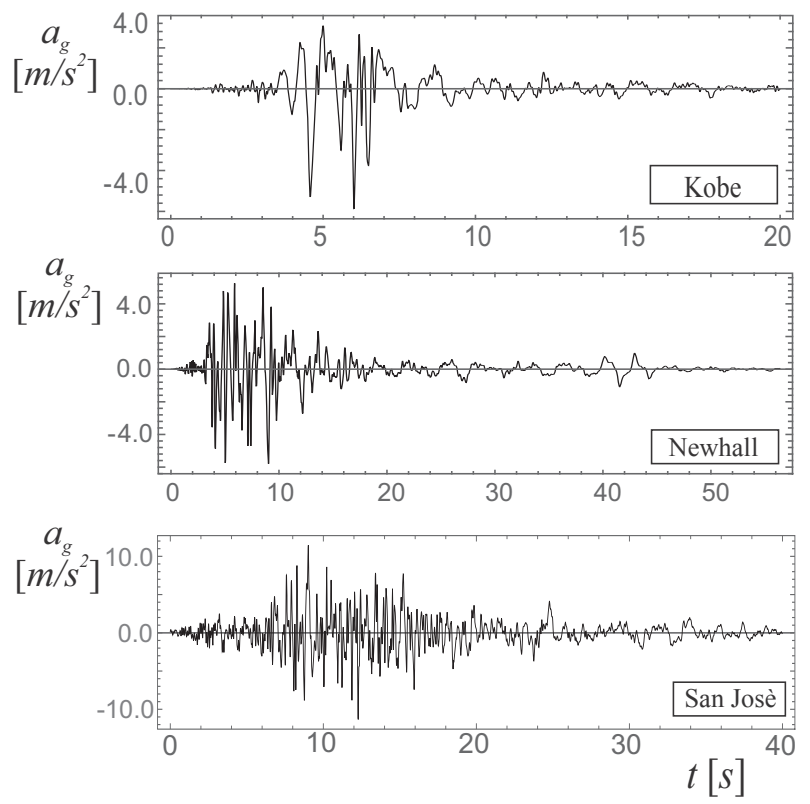

Figure 4: Time-histories of the recorded earthquakes.

The results of the parametric analysis performed with seismic excitation are arranged in rocking maps. Such rocking maps are contour plots of the maximum rocking angle in the parameter plane $2 b-\lambda$. The rocking maps obtained for rigid and deformable cabinets are compared to assess if the deformation improves the seismic behaviour of the cabinets.

In Fig. 5 the rocking maps are organized in matrix form where each column refers to a different earthquake (Kobe, Newhall, and San José). The first row shows the maps of the rigid cabinet, rows two and three refer to deformable cabinets with $T=1.0 \mathrm{~s}$ and $T=1.5 \mathrm{~s}$, respectively. In the maps, the light grey regions (rocking regions) are those where the cabinet undergoes rocking motion. The contour levels inside these regions refer to the maximum rocking angle reached during the motion. The dark grey regions (overturning regions) are those where the cabinet overturns. The first row of Fig. 5 shows that the rigid cabinet overturns in almost all the parameter plane except that in the right lower part of the maps that correspond to combinations of larger values of the base and lower slenderness of the cabinet. Rows two and three of Fig. 5 show that deformable cabinets have smaller overturning regions that generally tend to shrink as $T$ increases. As an exception, for the Newhall seismic event, the cabinet with $T=1.0 \mathrm{~s}$ does not overturn in the whole parameter plane, whereas the cabinet with $T=1.5 \mathrm{~s}$ overturns only in a small portion of the parameter space. Finally, the contour levels inside the light grey regions show a general reduction of the maximum rocking angle when the period $T$ increases. 
Kobe
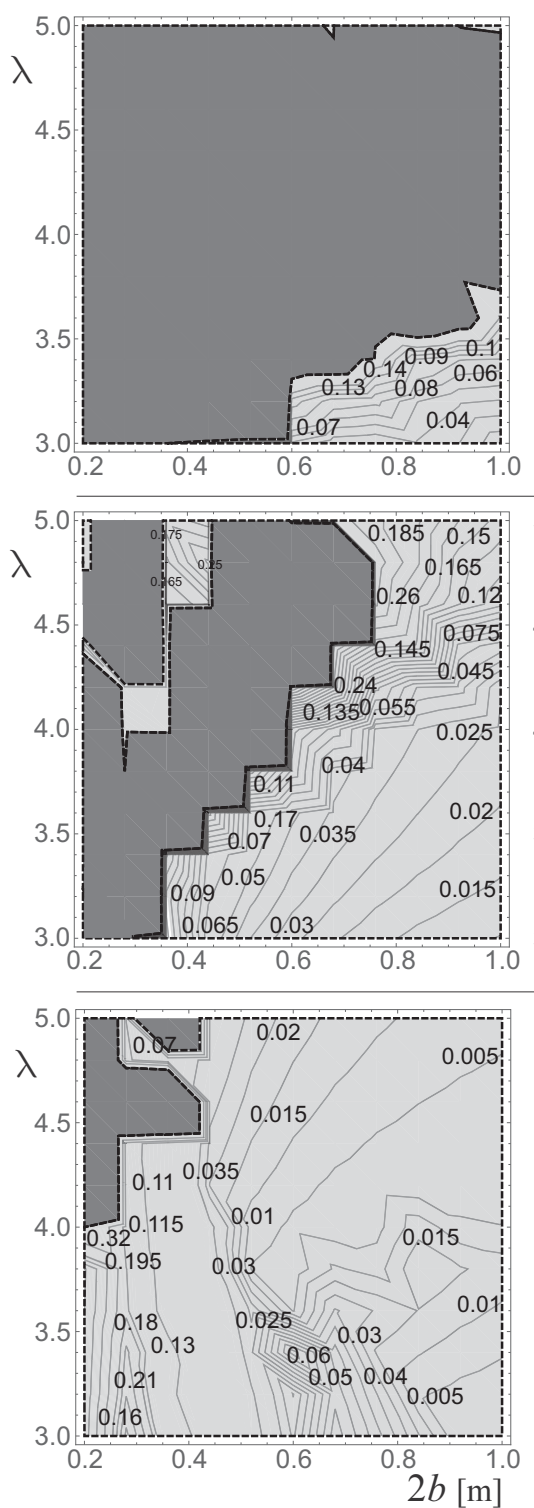

Newhall

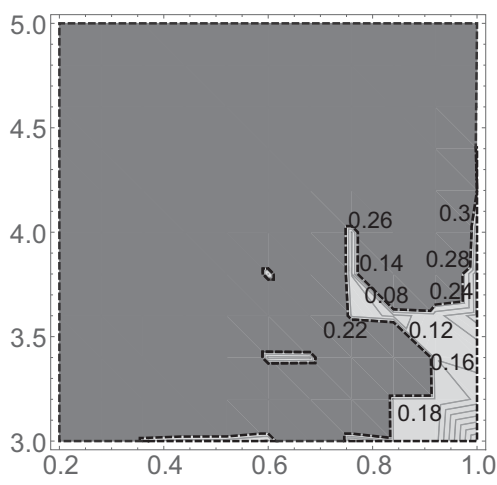

San Josè
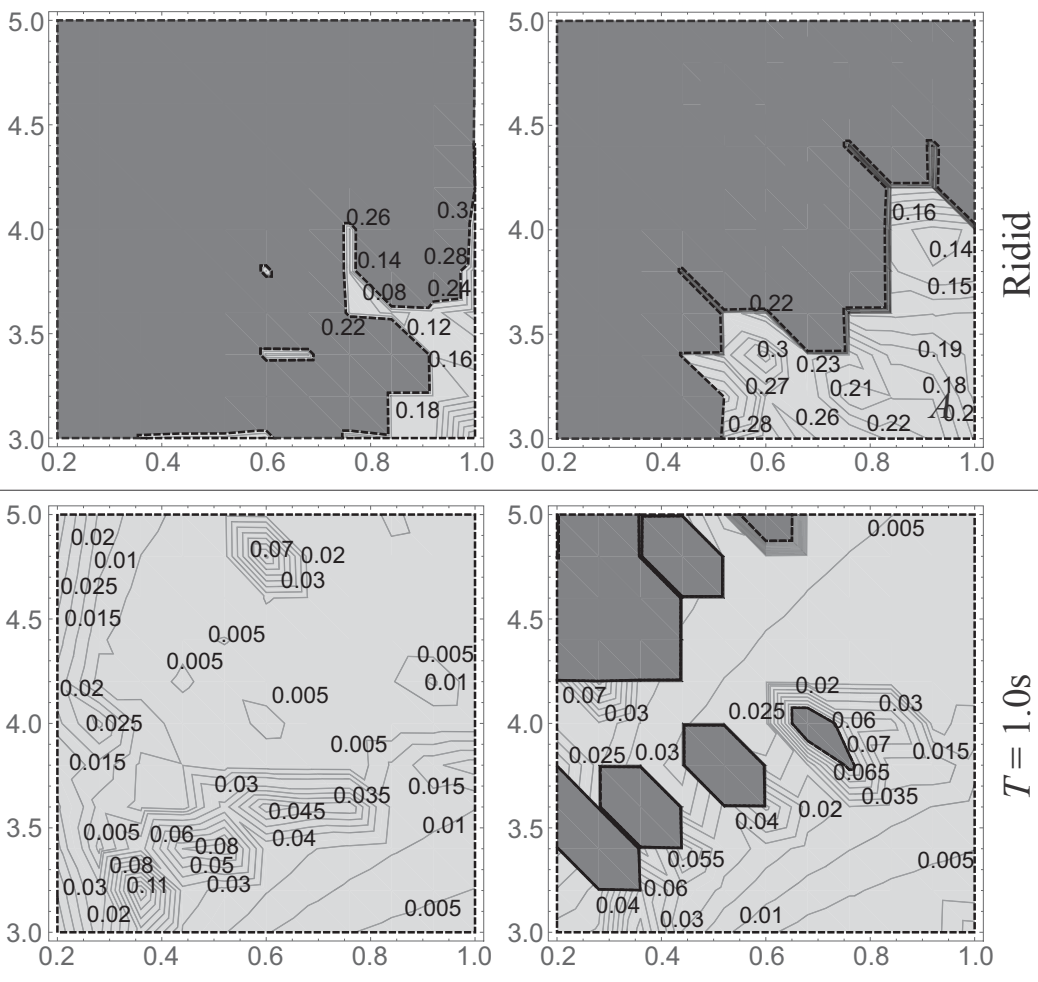
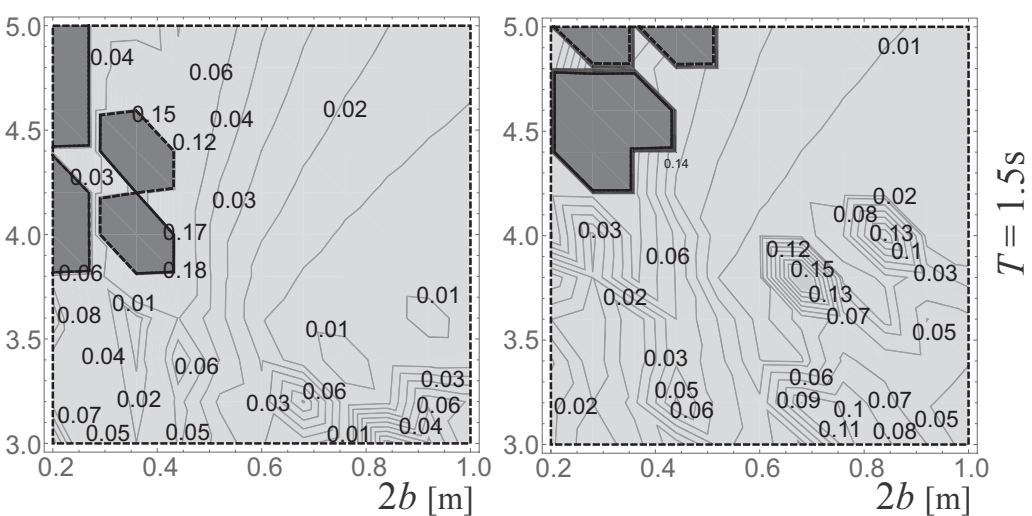

Figure 5: Rocking maps for three different earthquakes (Kobe, Newhall, San José) $\left(\xi=0.25, \eta=0.5, \theta_{\max }=0.1\right.$, cabinet full). 


\section{Conclusions}

This paper presents the model of a cabinet that can undergo a guided deformation. This model has been used to investigate the possibility to improve the dynamic and seismic behaviour of cabinets and racks by providing them of the ability to change their geometrical configuration during the rocking motion. The kinematic related to the change of geometrical configuration is specific to the model studied in this paper but can be easily generalized to other models representing cabinets and racks, both for civil and industrial use.

It has been assumed that the body can undergo only rocking motion, without sliding. The equations of motion have been obtained through a Lagrangian approach. The classical impact of the body base with the support during the rocking motion and the impact on security stops needed to limit the maximum angular deformation have been considered to describe the motion of the system.

An extensive parametric analysis has been performed by numerically integrating the equations of motion. The improvement in the dynamics has been analyzed by comparing the behaviour of rigid and deformable cabinets under one-sine pulse and seismic excitations. For the one-sine pulse excitation, the comparison has been made with overturning spectra that provide the amplitude of the excitation able to overturn the cabinets versus its circular frequency. For the seismic excitation, the maximum rocking angle reached during the motion under different recorded earthquakes have been arranged in rocking maps and compared.

The results shows that the ability of a cabinet to change its geometrical configuration before and after the start of the rocking motion significantly improves its dynamical and seismic performance compared to the performance of an equivalent rigid cabinet.

\section{References}

[1] A. Di Egidio and A. Contento. Base isolation of sliding-rocking non-symmetyric rigid blocks subjected to impulsive and seismic excitations. Engineering Structures, 31:27232734, 2009.

[2] A. Di Egidio and A. Contento. Seismic response of a non-symmetric rigid block on a constrained oscillating base. Engineering Structures, 32:3028-3039, 2010.

[3] A. Contento and A. Di Egidio. On the use of base isolation for the protection of rigid bodies placed on a multi-storey frame under seismic excitation. Engineering Structures, 62-63:1-10, 2014.

[4] I. Caliò and M. Marletta. Passive control of the seismic response of art objects. Engineering Structures, 25:1009-1018, 2003.

[5] M.F. Vassiliou and N. Makris. Analysis of the rocking response of rigid blocks standing free on a seismically isolated base. Earthquake engineering and structural dynamics, 41(2):177-196, 2012.

[6] N. Makris and J. Zhang. Rocking response of anchored blocks under pulse-type motions. Journal of Engineering Mechanics, 127(5):484-493, 2001.

[7] E.G. Dimitrakopoulos and M.J. DeJong. Overturning of retrofitted rocking structures under pulse-type excitations. Journal of Engineering Mechanics, 138:963-972, 2012. 
[8] L. Collini, R. Garziera, K. Riabova, M. Munitsyna, and A. Tasora. Oscillations control of rocking-block-type buildings by the addition of a tuned pendulum. Shock and Vibration, 2016:Article ID 8570538, 2016.

[9] P. Brzeski, T. Kapitaniak, and P. Perlikowski. The use of tuned mass absorber to prevent overturning of the rigid block during earthquake. International Journal of Structural Stability and Dynamics, 6(10):Article ID 1550075, 2016.

[10] A. de Leo, G. Simoneschi, C. Fabrizio, and A. Di Egidio. On the use of a pendulum as mass damper to control the rocking motion of a non-symmetric rigid block. Meccanica, 51:2727-2740, 2016.

[11] A. Di Egidio, R. Alaggio, A. Aloisio, A.M. de Leo, A. Contento, and M. Tursini. Analytical and experimental investigation into the effectiveness of a pendulum dynamic absorber to protect rigid blocks from overturning. Int. Journal of Non-Linear Mechanics, 115:1-10, 2019.

[12] G. Simoneschi, A. de Leo, and A. Di Egidio. Effectiveness of oscillating mass damper system in the protection of rigid blocks under impulsive excitation. Engineering Structures, 137:285-295, 2017.

[13] G. Simoneschi, A. Geniola, A. de Leo, and A. Di Egidio. On the seismic performances of rigid blocks coupled with an oscillating mass working as tmd. Earthquake Engineering and Structural Dynamics, 46:1453-1469, 2017.

[14] A. Di Egidio, A. de Leo, and G. Simoneschi. Effectiveness of mass-damper dynamic absorber on rocking block under one-sine pulse ground motion. International Limits of Non-Linear Mechanics, 98:154-162, 2018.

[15] R. Ceravolo, M.L. Pecorelli, and L. Zanotti Fragonara. Semi-active control of the rocking motion of monolithic art objects. Journal of Sound and Vibration, 374:1-16, 2016.

[16] R. Ceravolo, M.L. Pecorelli, and L. Zanotti Fragonara. Comparison of semi-active control strategies for rocking objects under pulse and harmonic excitations. Mechanical Systems and Signal Processing, 90:175-188, 2017.

[17] A. Di Egidio, G. Simoneschi, C. Olivieri, and A.M. de Leo. Protection of slender rigid blocks from the overturning by using an active control system. In Proceedings of the XXIII Conference The Italian Association of Theoretical and Applied Mechanics, 2014.

[18] G. Simoneschi, C. Olivieri, A.M. de Leo, and A. Di Egidio. Pole placement method to control the rocking motion of rigid blocks. Engineering Structures, 167:39-47, 2018.

[19] A. Di Egidio, C. Olivieri, and A.M. de Leo. Protection from overturning of rigid blocklike objects with linear quadratic regulator active control. Structural Control and Health Monitoring, 27, 2020.

[20] M. Michalis Vassiliou, K.R. MackieBozidar, and B. Stojadinovic. Dynamic response analysis of solitary flexible rocking bodies: Modeling and behavior under pulse-like ground excitation. Earthquake Engineering and Structural Dynamics, 43(10):1463-1481, 2014. 
[21] R. Thiers-Moggia and C. Málaga-Chuquitaype. Seismic control of flexible rocking structures using inerters. Earthquake Engineering and Structural Dynamics, 49(14):1519$1538,2020$.

[22] S. Acikgoz and M.J. DeJong. The interaction of elasticity and rocking in flexible structures allowed to uplift. Earthquake Engineering and Structural Dynamics, 41(15):2177- 2194, 2012.

[23] E. Cappelli, A. Di Egidio, and F. Vestroni. Analytical and experimental investigation into the behavior of a rocking masonry tuff wall. Journal of Engineering Mechanics, 146(6), 2020.

[24] J. Zhang and N. Makris. Rocking response of free-standing blocks under cycloidal pulses. Journal of Engineering Mechanics, 127(5):473-483, 2001.

[25] A. Di Egidio, A.M. de Leo, and A. Contento. The use of a pendulum dynamic mass absorber to protect a trilithic symmetric system from the overturning. Mathematical Problems in Engineering, 2019, article Id: 4843738:14, 2019. 